\title{
Design and Synthesis of Ruthenium Oligothienylacetylide Complexes: New Materials for Acoustically Induced Nonlinear Optics
}

\section{Supporting Information}

\section{DFWM technique and theoretical approach.}

To measure the third-order susceptibilities of the studied organomettalic compounds, we have used the degenerate four wave mixing technique. The geometry of the experimental arrangement is presented in fig.1. $30 \mathrm{ps}$ laser pulses at $\lambda=532 \mathrm{~nm}$ provide the excitation generated by an amplified mode locked Quantel Nd: YAG laser operating at $1 \mathrm{~Hz}$ repetition rate. Two of the interacted beams are strong counterpropagating pumps travelling in the forward and backward directions. Their intensities verify the relation $I_{1}(z=0)=I_{2}(z=\ell)$. The third input wave is a weak probe $\left(\mathrm{I}_{3}=10^{-2} \mathrm{I}_{1}\right)$ which makes an angle of $12^{\circ}$ with respect to the pump wave direction. The signal wave $<4>$ is radiated in backward direction of the probe beam.

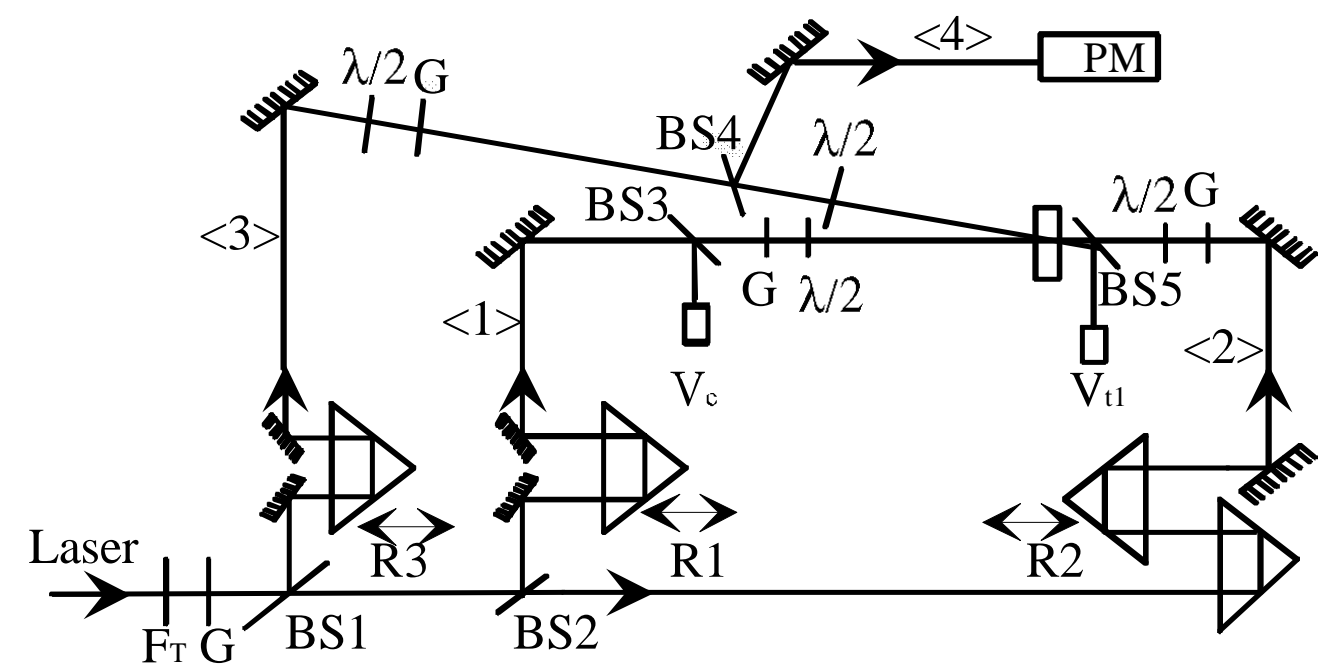

Fig. 1. The schematic diagram of the experimental set-up of DFWM : $\langle 1\rangle,\langle 2\rangle$ - are pump beams; $<3>$ probe beam; $<4>$ fourth beam or signal; $F_{T}$ neutral filter; $R_{1}-R_{3}$ delay lines; $G$ glan prism; $\mathrm{V}_{\mathrm{c}}$ and $\mathrm{V}_{\mathrm{T} 1}$ control photodiodes; $\mathrm{P}_{\mathrm{M}}$ photomultiplier tube; $\mathrm{BS} 1,2,3,4$ beam splitter. 
The theoretical interpretation of DFWM is based on the Maxwell nonlinear propagation equations. Using slowly varying envelope approximation we obtain DFWM efficiency in the form. ${ }^{1}$

$$
\begin{array}{ll}
\mathrm{R}=\frac{\mathrm{I}^{<4>}(0)}{\mathrm{I}^{<3>}(0)}=\{ & \mathrm{p}^{2} \geq 0 \\
\frac{\mathrm{p}^{2}+\frac{\alpha^{2}}{4}}{\left[\mathrm{p}\left(\operatorname{ctg}(\mathrm{p} \ell)+\frac{\alpha}{2}\right]^{2}\right.} & \mathrm{p}^{2}<0 \\
\text { where } \quad \mathrm{p}^{2}=\left(\frac{\alpha^{2}}{n^{2} c \lambda} \chi^{<3>}\right)^{2} \mathrm{I}_{1}(0)^{2} \exp (-\alpha \ell)-\frac{\alpha^{2}}{4}, & \mathrm{q}=i \mathrm{p} .
\end{array}
$$

The parameters $\alpha, \ell, \lambda, n$ and $c$ are respectively the linear absorption coefficient, the cell length, the wavelength of the laser, and the linear refractive index of the material and the velocity of light propagation in vacuum. The absolute value of $\chi^{\langle 3\rangle}$ can be obtained by adjusting the theoretical curve given by the formula (1) to the experimental data of R.

As all the compounds are studied in solution, an increase of the concentration leads to an increase of the absorption so we found a compromise between optical nonlinearties and optical losses. For this purpose, we measure the influence of concentration on signal $\mathrm{I}^{\langle 4\rangle}$ for each compound. We observe that all compounds display the same behavior: a single maximum of $\mathrm{I}^{<4>}$ that corresponds to an optimum value of concentration $\mathrm{C}_{\mathrm{opt}}$. We mesure the efficiency of DFWM for each compound at $\mathrm{C}_{\mathrm{opt}}$. The transmission measurements at the small concentrations and for the range of energy intensity used $\left(0-1.2 \mathrm{GW} / \mathrm{cm}^{2}\right)$, show that all molecules studied present a very weak linear absorption. The $\mathrm{C}_{\mathrm{opt}}$ values and the absorption coefficients at $\mathrm{C}_{\text {opt }}$ deduced from transmission measurements are collected in Table 4 .

The reflectivity $\mathrm{R}$ at $\mathrm{C}_{\mathrm{opt}}$ was measured as a function of the wave $<1>$ intensity for the vertical polarisation of incident beams. The studied solutions present the same behavior. We observe a good agreement between experimental results and the theoretical curve given by the

${ }^{1}$ (a) Sahraoui, B. ; Rivoire G. Opt. Comm. 1997, 138, 109. (b) Sahraoui, B.; Nguyen Phu, X.;

Sallé, M.; Gorgues, A. Opt. Lett., 1998, 23, 1811. 
formula (1). The adjustment of the theoretical curve to experimental data allows us to deduce the susceptibility values $\chi^{\langle 3\rangle}$ for the studied compounds. The results are collected in the Table 4. In order to characterise the nonlinear optical nonlinearities of individual molecule we determine the second order hyperpolarisability using the following relation

$$
\chi^{<3>}=\mathrm{F}^{4} N \gamma_{\text {solution }}+\chi_{\text {solvent }}^{<3>}
$$

where $\mathrm{F}=\left(\mathrm{n}^{2}+2\right) / 3$ is the Lorentz field factor correction, $N=\mathrm{N}_{\mathrm{A}} \cdot \mathrm{C} / \mathrm{M}$ is number of solution

molecules per the unity of volume; $\mathrm{N}_{\mathrm{A}}$ is Avogadro number. Since $\chi_{\text {solvent }}^{<3>}$ is small, it can be neglected. The second order hyperpolarisability values are given in Table 4 .

\section{AIOSHG measurements}

The compounds were incorporated into the PMMA matrix. The investigated samples were prepared as a mixture of $4 \mathrm{~g}$ of PMMA and $0.035 \mathrm{~g}$ of chromophore and dissolved in $30 \mathrm{ml}$ of chloroform in the argon atmosphere. This mixture was prepared using a magnetical stirrer and they were put in the Alumina crucible after that. Verifying the evaporation rate after the 24 hours, we have obtained samples we are investigating here. One of the complications of the complexes investigated is their photodestruction under the influence of the laser light. Specially performed NMR and IR investigations have shown that under the influence of the laser light, particularly of the power density higher than the $450 \mathrm{MW} / \mathrm{cm}^{2}$ a partial destruction of the chromophore has occurred, however in the PMMA matrix this degradation does not exceed $7.2 \%$, and the major part of the molecule participated in the processes desirable.

The principal measurement set-up for the AIOSHG measurements is depicted in the Fig. 2. A pulsed mode-locked $\mathrm{Dy}^{3+}$ :YAB laser $(\lambda=1.76 \mu \mathrm{m}$; power about $25 \mathrm{MW}$; pulse duration about $15 \mathrm{ps)} \mathrm{was} \mathrm{used} \mathrm{as} \mathrm{a} \mathrm{fundamental} \mathrm{laser} \mathrm{beam} \mathrm{for} \mathrm{optical} \mathrm{second} \mathrm{harmonic} \mathrm{generation.} \mathrm{The}$ acoustic field was applied to the investigated composites, which were in form of plates with thickness ranging within $1.4 \mathrm{~mm}$ using $\mathrm{LiNbO}_{3}$ piezoelectric acoustical transducer (AT), which allows varying acoustical frequencies $(80 \mathrm{~Hz}-1.1 \mathrm{MHz})$. The AT was attached to the 
surface of the sample. Transverse acoustic wave excited by transverse $\mathrm{c}_{66}$ stiffness component of $\mathrm{LiNbO}_{3}$ was used. The transducer is attached directly on the sample's surfaces by a method of optical contact perpendicularly to the beam propagation direction. The investigated samples have a form of parallelepiped with dimensions $3 \times 3 \times 0.7 \mathrm{~mm}$. The absorbance of the acoustic wave was monitored by echo method and do not exceed $0.1 \mathrm{~dB}$. To avoid standing wave resonances at low frequencies we have done the walls of the samples slightly non-parallel to avoid creation of the resonance-like effects.

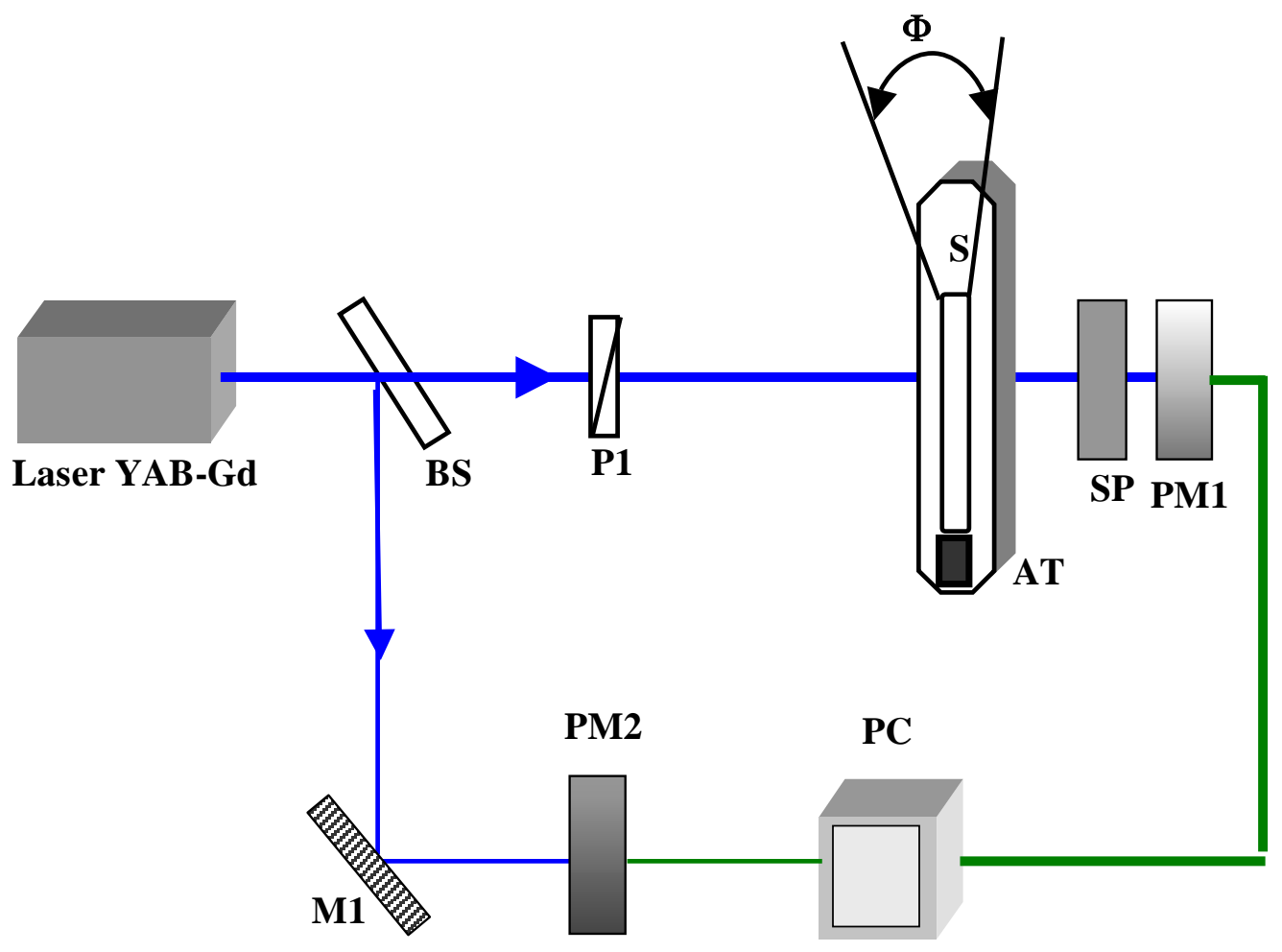

Fig. 2. Principal schema of the AIOSHG experiment. BS-beamsplitter; M1, M2 - mirrors; P1, P2-polarisers; AT - acoustical transducers; $\mathbf{S}$ - specimens kept in liquid helium cryostat; SP-spectrograph; PM1,PM2 -photomultipliers; PC - personnel computer. 
The set-up allows the transfer of up to $46 \%$ of the acoustic power to the composite. The angle between the composite surface and incident beam direction was changed in order to obtain the angle dependencies of the AISHG within the Maker fringence scheme. ${ }^{2}$

The incident laser beam was polarized using polarizers P1, P2 (with degree of polarization of about $99.997 \%$ ) and the output doubled-frequency AIOSHG light intensities were detected using photomultiplier PM1 connected with 690 ps gate electronic boxcar. The short-time pulse duration allowed avoiding the increase of local temperature. Laser beam was spatially filtered in order to obtain a homogenous light profile (usually of the Gaussian-like shape profile sequence) possessing the per-pulse-energy power densities varying within the $0.6-1.2 \mathrm{GW} / \mathrm{cm}^{2}$ range. Grating spectrophotometer SP separated fundamental beam of $\mathrm{Gd}^{3+}:$ YAB laser $(\lambda=1.76 \mu \mathrm{m})$ from the AIOSHG $(\lambda=0.88 \mu \mathrm{m})$ doubled-frequency optical beam. For incident and output beam energies monitoring photomultipliers PM1 and PM2 connected to the $\mathbf{P C}$ were used.

During evaluation of the second-order nonlinear optical response we measured light intensities $I$ for the $\omega$ and $2 \omega$ laser frequencies. The evaluation of the nonlinear optical susceptibility was performed using a method described in Ref. 3.

The calculations were corrected by taking into account the angle-dependent birefringence $\Delta \mathrm{n}_{\theta}$ and changes of thickness $\mathrm{d}_{\theta}$ by using the following expression:

$$
I=I_{0} \sin ^{2}\left(\pi \Delta n_{\theta} d_{\theta} / \lambda\right)
$$

where $I$ and $I_{0}$ are the output and incident light intensities, respectively. Polarization of the optical beam was parallel to the polarization of the transverse acoustic wave polarization for every angle $\theta$ between laser beam propagation and composite's surface.

\footnotetext{
${ }^{2}$ Maker, P.D.; Terhune, R.W.; Nisenoff M.and Savage, C.M. Phys. Rev. Lett., 1962, 8, 41.

${ }^{3}$ Kityk, I.V.; Zmija, J.; Majchrowski, A.; Ebothe. J. J. Appl. Phys. 2003, 93, 2, 1160.
} 
To detect the possible phase transitions we used DSC (Differential Scanning Calorimetric) method using a Netzsch STA 406C DSC instrument with low temperature Controller -TASC 414/3 (Netzsch). Powder like samples were put in an $\mathrm{Al}_{2} \mathrm{O}_{3}$ crucible and cooled from ambient temperature to $4.2 \mathrm{~K}$ in argon atmosphere with a rate of $0.18 \mathrm{~K}$ per minute and thermostabilisation of up to $0.1 \mathrm{~K}$. Measurements were performed both during cooling and heating to achieve additional control of ferroelectric spontaneous polarization $\mathrm{P}_{\mathrm{s}}$ The traditional Tower-Sower method gave the possibility to evaluate the $\mathrm{P}_{\mathrm{s}}$ with precision of up to $0.5 \mu \mathrm{C} / \mathrm{cm}^{2}$. The samples were controlled by Raman spectra using $\operatorname{Kr}$ laser $(\lambda=745$ $\mathrm{nm})$ as a source. 